\title{
Reconfiguration time and complexity minimized trust-based clustering scheme for MANETs
}

\author{
Sunho Seo ${ }^{1}$, Jin-Won Kim ${ }^{1,2}$, Jae-Dong Kim ${ }^{1,2}$ and Jong-Moon Chung ${ }^{1 *}$ (D)
}

\begin{abstract}
A trust management mechanism for mobile ad hoc networks (MANETs) is proposed to cope with security issues that MANETs face due to time constraints as well as resource constraints in bandwidth, computational power, battery life, and unique wireless characteristics. The trust-based reputation scheme GlobalTrust is a reliable trust management mechanism. In this paper, a clustering algorithm is applied to the GlobalTrust scheme (named Cluster-based GlobalTrust (CGTrust)) to find the optimal group size to minimize the configuration time, which consists of trust information computational time and complexity, while having to satisfy the trust reliability requirements. The optimal number of clusters is derived from the minimizing point of the computation complexity function. Simulation results show that the computational time and complexity of CGTrust are controllable and can be used effectively in time critical network operations that require trust analysis.
\end{abstract}

Keywords: MANET, Trust, Cluster, GlobalTrust, CGTrust

\section{Introduction}

Mobile ad hoc networks (MANETs) consist of distributed wireless mobile nodes. In MANETs, it is critical to make a decision of assessing the trustworthiness of participating nodes accurately by a trust authority. Trust evaluations are made on node reputation, which is the perception of a node evaluated by other nodes. A node's reputation is evaluated based on the collection of trust evaluations made on that node by other nodes [1, 2]. Cho et al. [1] analyzed the concepts and properties of trust. In addition, they surveyed MANET trust management schemes for secure routing, authentication, and intrusion detection, etc. Aberer and Despotovic [3] proposed a trustbased reputation management scheme. The authors of $[4,5]$ proposed distributed methods for reputation management. However, results in [3-5] are vulnerable to collusive attacks. Reputation management schemes in [6-9] subjectively evaluate reputation using direct observation that disturbs the global view. Quorum-based reputation management schemes are proposed based on k-out-of- $n$

\footnotetext{
*Correspondence: jmc@yonsei.ac.kr

${ }^{1}$ School of Electrical and Electronic Engineering, Yonsei University, Seoul,

Republic of Korea

Full list of author information is available at the end of the article
}

threshold signatures $[10,11]$. The $k$-means-based reputation scheme is presented in $[12,13]$, which does not consider conflicting recommendation attacks. Chen et al. [2] proposed GlobalTrust for accurate decision-making of a trusted authority (TA) under various attacks, which shows an outstanding performance in trust on MANETs. The TA needs to collect trust-related data and compute trust values and set up optimal routing paths, which is a very computationally burdening task. Thus, a common issue would be the time constraints and required computing resources of the TA and MANET nodes. The computational time delay and complexity of GlobalTrust significantly increases as the number of nodes in the MANET increases. In addition, in a MANET, communicating nodes are mobile. Therefore, route reconfiguration may need to be conducted frequently. As a result, reconfiguration time minimization would be necessary. In this paper, a Cluster-based GlobalTrust (CGTrust) scheme is proposed with the objective to minimize the computational complexity and reconfiguration time delay experienced by a TA while supporting the required trust reliability requirements. CGTrust consists of the following three unique features. 
- CGTrust evaluates trust at cluster heads (CHs) and at the TA in contrast to GlobalTrust that focuses on the TA. This approach has the benefit of requiring less time and uses less computing resources at the TA in the network.

- CGTrust provides a computational complexity minimized mechanism to form MANET clustering, where the complexity considers both intra and inter cluster computations. This algorithm helps to drastically reduce the complexity associated with the trust evaluation and computation.

- CGTrust provides a mechanism to evaluate the trust of both non- $\mathrm{CH}$ nodes and $\mathrm{CH}$ s to prevent false trustworthiness decisions of non- $\mathrm{CH}$ nodes by a non-trustworthy $\mathrm{CH}$ and minimize the setup and reconfiguration time.

\section{CGTrust}

The proposed scheme is for MANETs that use the GlobalTrust trust-based reputation scheme [2]. It is assumed that MANETs consist of multiple nodes communicating via multiple hops. The objective of CGTrust is to provide a trust-based clustering algorithm that derives the optimal number of clusters $k$ (i.e., $k_{\text {optimal }}$ ) to minimized network setup and reconfiguration time delay based on the constraints of the required trust level that needs to be satisfied. CGTrust forms clusters by using a trust-based reputation algorithm, where the TA makes decisions based on trust values. The TA is a system that can determine the trust level of all nodes in the network and manage the security and performance of the network [2]. Therefore, it is assumed that the TA does not perform the role of a node in the network, rather the TA exists independently and manages the network because the TA requires a large amount of resources to perform the trustworthiness evaluation functions. The TA collects evidence periodically to assess the trust of participating nodes through $\mathrm{CHs}$ and makes a trustworthy decision. In the initial setup stage, if no $\mathrm{CH}$ s have been preselected, then the TA will select $\mathrm{CHs}$ and provide initial cluster information (where this initial formation may not be an optimal setting). If The number of nodes changes, the TA recomputes the optimal number of clusters, and then reassigns $\mathrm{CHs}$ among the nodes with the highest reputation values. Figure 1 is a diagram

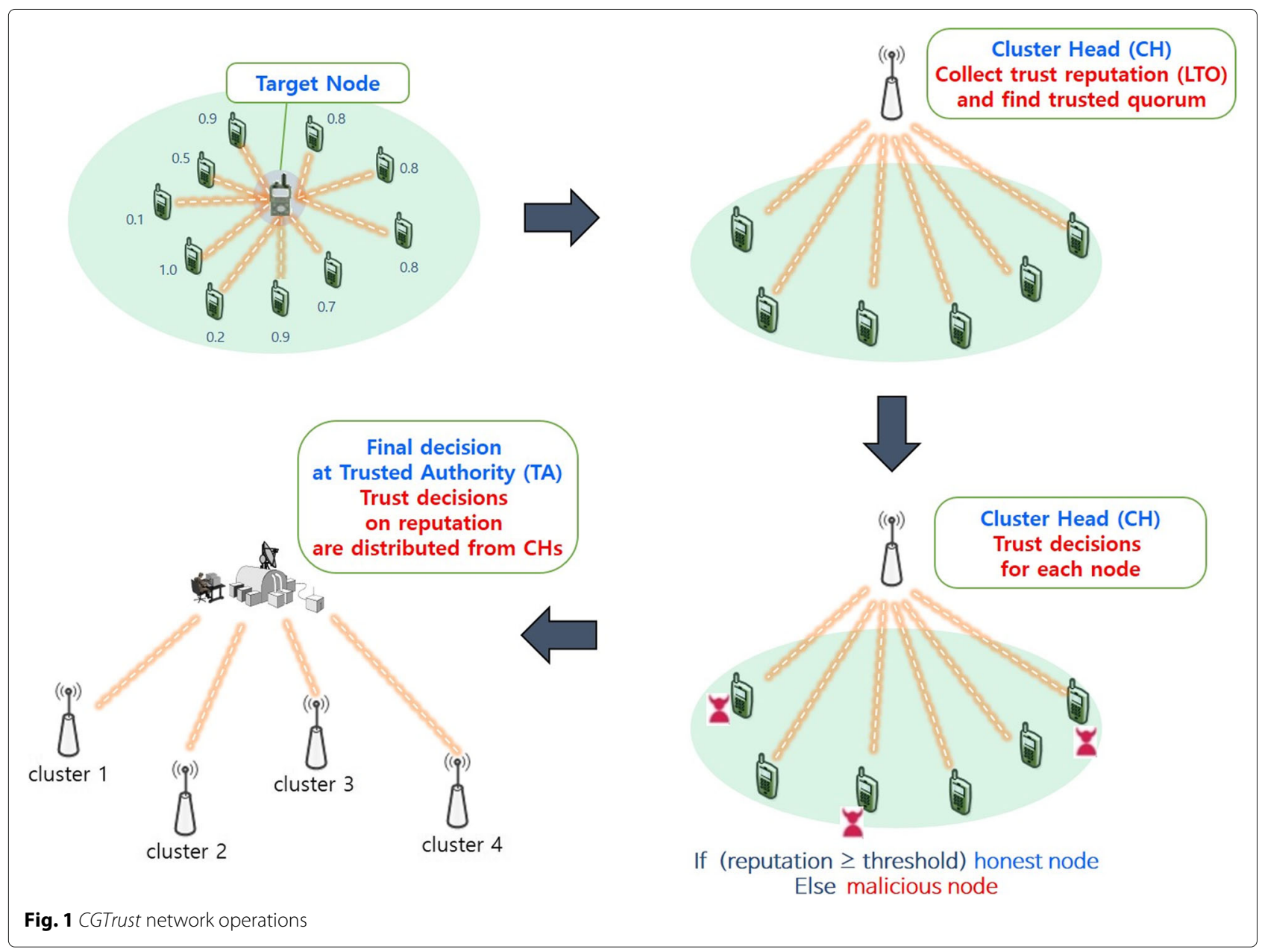


that illustrates the operations of CGTrust. The CGTrust process is described in the following steps (Fig. 2).

Step 1: Non-CH nodes that belong to the $i$ th cluster transmit local trust opinions (LTOs) of their neighbor nodes to the local $\mathrm{CH}$. $\mathrm{LTO}_{w, u}$ is node $w$ 's trust opinion towards node $u$ based on direct observations. $\mathrm{LTO}_{w, u}$ is calculated as below

$$
\mathrm{LTO}_{w, u}=\frac{p_{w, u}}{p_{w, u}+n_{w, u}}
$$

where $p_{w, u}$ and $n_{w, u}$ are respectively the total number of positive events and the total number of negative events. If there are no events between node $w$ and node $u$, the LTO is null. The sum of positive and negative events $\left(p_{w, u}+n_{w, u}\right)$ is the sum of the number of events sent by node $w$ to node $u$. Positive events represented by $p_{w, u}$ are events that node $w$ is determined that a packet was transmitted well to node $u$. Negative events represented by $n_{w, u}$ are events that node $w$ is determined that a packet was not delivered to node $u$ for some reasons (noise, wrong decision, intentional packet drop, etc.). The transmission can be determined by an acknowledgement packet, etc. As can be seen from Eq. (1), the higher the positive events, the closer to 1 the LTO and the closer to 0 the negative events become. The higher the LTO, the trust opinion of node $u$ approaches the reputation of an honest node.

An honest node correctly sends a packet to a predefined route when it receives a packet from another node, except for errors that occur during the transmission process. In

\section{Algorithm: CGTrust \\ At the $\mathrm{CH}-$ Compute trust of the cluster \\ 1: COMPUTE local trust opinion LTO \\ 2: COMPUTE Sim and SR \\ 3: FIND CH's trusted quorum $D$ \\ 4: COMPUTE $B R, C R$ and $G R$ \\ At the TA-Compute trust of all nodes \\ 5: IF cluster is using encrypted packet mode $(\beta)$ COLLECT encrypted LTOs from non-CH nodes \\ 6: ELSE \\ COLLECT $G R$ from $\mathrm{CH}$ \\ 7: COMPUTE Sim and SR \\ 8: FIND TA's trusted quorum $D$ \\ 9: COMPUTE $B R, C R$, and $G R$ \\ 10: IF $G R$ is unknown \\ SET the trust level of the node as unknown \\ 11: ELSEIF $G R \geq$ decision factor $\theta$ \\ SET the trust level of the node as a honest node \\ 12: ELSE \\ SET the trust level of the node as a malicious node \\ 14: IF $N$ has changed \\ COMPUTE $k_{\text {optimal }}$ using (15) \\ Assign nodes to the $k_{\text {optimal }}$ clusters and select $\mathrm{CHs}$}

Fig. 2 CGTrust algorithm addition, when the LTO is reported to the TA or $\mathrm{CH}$, the reporting of the LTO is performed without distortion.

A malicious node may drop a packet when it is received from another node, or intentionally send a packet to a different route. In addition, information may be distorted during the transmission and reporting process of the LTO to the $\mathrm{TA}$ or $\mathrm{CH}$.

The $i$ th $\mathrm{CH}\left(\mathrm{CH}_{i}\right)$ aggregates trust-related evidences and computes the subjective reputation (SR) of node $u$ as it is assessed by node $w\left(\mathrm{SR}_{w, u}\right)$ using

$$
\mathrm{SR}_{w, u}=\sum_{j \in S_{u}} \mathrm{LTO}_{j, u} \frac{\mathrm{HR}_{j} \operatorname{Sim}(w, j)}{\sum_{j \in S_{u}} \mathrm{HR}_{j} \operatorname{Sim}(w, j)}
$$

where $\operatorname{Sim}(w, j)$ is the cosine similarity of the LTOs between node $w$ and node $j, S_{u}$ is the set of nodes that have non-null LTOs over node $u$ (including $w$ if $w$ has one), and $\mathrm{HR}_{j}$ is the hierarchical rank of node $j . \mathrm{HR}_{j}$ can specify that administrators have different values, or they can all have the same value, depending on how trustworthy the node's opinion is [2]. The TA should have a hierarchy rank higher than that of any $\mathrm{CH}$ or node, because the TA's opinion is more strongly reflected than the $\mathrm{CHs}$ or nodes as it is where the reputation judgment is made. In this paper, the hierarchy rank is designated as $\mathrm{TA}=3, \mathrm{CH}=2$, and node $=1$.

After calculating the $\mathrm{SR}, \mathrm{CH}_{i}$ makes a SR matrix. The SR tuple in node $w$ 's view is denoted as a vector. $\mathrm{CH}_{i}$ compares all SR tuples, and merges the two SR tuples with the least difference based on the agglomerative hierarchical clustering technique [2]. $\mathrm{CHs}$ use this technique to form its trusted quorum $\left(D_{C H}\right)$. The process of finding the trusted quorum ends when the set having the largest number of nodes has more than half of the total number of network nodes. When the total number of nodes is $N$, the number of nodes belonging to the trusted quorum may have a value between $[N / 2, N]$, depending on how it is calculated. Since all SR tuples are compared and a trusted quorum is found, the complexity does not change depending on the size of the trusted quorum.

Based on the trusted quorum, $\mathrm{CH}_{i}$ obtains the behavioral reputation (BR) of node $u\left(\mathrm{BR}_{\mathrm{CH}_{i, u}}\right) . \mathrm{BR}_{\mathrm{CH}_{i, u}}$, reflecting how $\mathrm{CH}_{i}$ views node $u$ 's network behavior, is computed by averaging the SR tuples using (3).

$$
\mathrm{BR}_{\mathrm{CH}_{i, u}}=\frac{\sum_{w \in D} \mathrm{SR}_{w, u}}{\left|D_{C H}\right|}
$$

Then $\mathrm{CH}_{i}$ computes the credibility reputation (CR) of node $u\left(\mathrm{CR}_{\mathrm{CH}_{i, u}}\right)$ using (4). $\mathrm{CR}_{\mathrm{CH}_{i, u}}$ indicates how trustworthy $u$ 's reported LTOs are. This is computed based on 
the difference between $u$ 's reported LTOs and BRs of the nodes that node $u$ has reported LTOs over.

$$
\begin{aligned}
& \mathrm{CR}_{\mathrm{CH}_{i, u}}=
\end{aligned}
$$

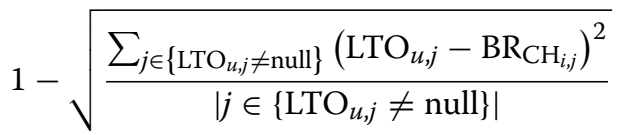

Next $\mathrm{CH}_{i}$ computes the global reputation (GR) of node $u\left(\mathrm{GR}_{\mathrm{CH}_{i, u}}\right)$ using the normalized factor $\gamma$ selected from the range in $[0,1]$.

$$
\mathrm{GR}_{\mathrm{CH}_{i, u}}=\gamma \mathrm{BR}_{\mathrm{CH}_{i, u}}+(1-\gamma) \mathrm{CR}_{\mathrm{CH}_{i, u}}
$$

Step 2: Once a $\mathrm{CH}$ computes all GRs of its cluster's nodes, it sends the result information to the TA. The TA computes the SR from GR based on the resultant data as in (6). The cosine similarity $\left(\operatorname{Sim}\left(\mathrm{CH}_{i}, \mathrm{CH}_{k}\right)\right)$ in Eq. (6) is calculated on the basis of the LTO between the $\mathrm{CH}$ communication. This allows the TA to determine if a $\mathrm{CH}$ is infected and can make a final decision. Step 2 is performed when the number of clusters is two or more. If the network is not divided into clusters, the TA directly makes all trust decisions of all nodes in the network.

$$
\begin{aligned}
& \sum_{\mathrm{CH}_{k} \in S_{u}^{\prime}} \mathrm{GR}_{\mathrm{CH}_{i, u}}= \\
& \mathrm{CR}_{k}, u \frac{\mathrm{HR}_{\mathrm{CH}_{k}} \operatorname{Sim}\left(\mathrm{CH}_{i}, \mathrm{CH}_{k}\right)}{\sum_{\mathrm{CH}_{k} \in S_{u}^{\prime}} \mathrm{HR}_{\mathrm{CH}_{k}} \operatorname{Sim}\left(\mathrm{CH}_{i}, \mathrm{CH}_{k}\right)}
\end{aligned}
$$

After (6), the TA calculates the BR of node $u$ in (7), and the $\mathrm{CR}$ of node $u$ (i.e., $\mathrm{CR}_{\mathrm{TA}, u}$ ) in (8).

$$
\mathrm{BR}_{\mathrm{TA}, u}=\frac{\sum_{\mathrm{CH}_{i} \in D} \mathrm{SR}_{\mathrm{CH}_{i, u}}}{\left|D_{\mathrm{TA}}\right|}
$$

$$
\begin{aligned}
& \mathrm{CR}_{\mathrm{TA}, u}= \\
& 1-\sqrt{\frac{\left.\sum_{u \in\left\{\mathrm{GR}_{\mathrm{CH}_{i, u}} \neq \mathrm{nulll}\left(\mathrm{GR}_{\mathrm{CH}}-u\right.\right.}-\mathrm{BR}_{\mathrm{TA}, u}\right)^{2}}{\mid u \in\left\{\mathrm{GR}_{\mathrm{CH}_{i, u}} \neq \text { null }\right\} \mid}}
\end{aligned}
$$

Then the TA computes the GR of node $u$ (i.e., $\mathrm{GR}_{\mathrm{TA}, u}$ ) using

$$
\mathrm{GR}_{\mathrm{TA}, u}=\gamma^{\prime} \mathrm{BR}_{\mathrm{TA}, u}+\left(1-\gamma^{\prime}\right) \mathrm{CR}_{\mathrm{TA}, u}
$$

Step 3: After the TA computes the GR, the TA decides the trustworthiness of each node using

$$
\begin{aligned}
& \operatorname{Decision}(u)= \\
& \left\{\begin{array}{l}
\text { unknown if } \mathrm{GR}_{\mathrm{TA}, u}=\text { unknown } \\
\text { honest if } \mathrm{GR}_{\mathrm{TA}, u} \geq \theta \\
\text { malicious if } \mathrm{GR}_{\mathrm{TA}, u}<\theta
\end{array}\right.
\end{aligned}
$$

where $\theta$ is a decision factor selected from the range in $[0,1]$. The detection errors can be reduced by selecting the most appropriate $\theta$ value.

The TA evaluates each CH's trustworthiness using direct trust computation, which can be conducted using the encrypted packet mode, which encrypts packets exchanged between non- $\mathrm{CH}$ nodes and the TA. In this mode, non- $\mathrm{CH}$ nodes send (encrypted) information packets to their $\mathrm{CH}$ and the $\mathrm{CH}$ forwards these packets to the TA without trust computation. Using the encrypted packet mode, the TA computes the CHs' trustworthy level periodically considering $\beta$, which is the ratio of nodes that use encrypted packet mode in the cluster. In this computation process, $\beta$ is a variable that represents the possibility that a $\mathrm{CH}$ is a malicious node. As the value of $\beta$ increases, the number of nodes that the TA needs to directly compute increases, making it difficult to reduce the computational complexity. Considering the computational complexity of trust computation and the worst case where the majority of $\mathrm{CHs}$ are infected, the suitable value of $\beta$ is $[0,0.5]$.

\section{Cluster-based network analysis}

\subsection{Computational complexity analysis}

The proposed scheme computes the GR of each node based on GlobalTrust that uses a trusted quorum $D$. GlobalTrust uses the agglomerative hierarchical clustering technique to find a minimum dominating cluster [2].

For an accurate complexity analysis, the method of [14] is applied to CGTrust, where the complexity of the pseudocode steps is computed. Each cluster has $N / k$ nodes. Every node will collect the SRs of all other nodes in its cluster, which are $((N / k)-1)$ SRs. Two nodes in a cluster will pair up and compare their collected SRs, but will exclude the SR of the paired node in this comparison process. Therefore, $((N / k)-2)$ SRs will be compared by the node pair. In addition, since there are $\left(\begin{array}{c}N / k \\ 2\end{array}\right)$ combinations of possible node pairs in each cluster, the computational complexity of one cluster is $\mathrm{O}[((N / k)-2)(N / k)((N / k)-1) / 2]$ $=\mathrm{O}\left((N / k)^{3}\right)$ (step 3$)$.

In case of inter-clusters, the TA uses $((1-\beta) k+\beta N-1)$ SRs for each node since the TA computes the GR directly at the rate of $\beta$. The minimum pair complexity becomes $\mathrm{O}\left(((1-\beta) k+\beta N-1)^{2}\right)$. The computational complexity for all steps is $\mathrm{O}(((1-\beta) k+\beta N-1)((1-\beta) k+\beta N-2) / 2))$ and based on this the computational complexity of each node is $\mathrm{O}\left(((1-\beta) k+\beta N-1)^{2}\right)+\mathrm{O}(((1-\beta) k+$ $\beta N-1)((1-\beta) k+\beta N-2) / 2))$. Therefore, to compute the TA's trusted quorum the required complexity is $\mathrm{O}\left[((1-\beta) k+\beta N-1)^{2}+((1-\beta) k+\beta N-1)((1-\beta)\right.$ $k+\beta N-2) / 2)(1-\beta) k+\beta N]$ (step 9). After organizing the terms, the computational complexity can be expressed as $\mathrm{O}\left((1-\beta)^{3} k^{3}+(\beta N)^{3}\right)$. In CGTrust, the complexity of both intra-cluster and inter-clusters are considered. Therefore, the total computational complexity $\left(C_{\text {total }}\right)$ becomes

$$
C_{\text {total }}=O\left((N / k)^{3}+(1-\beta)^{3} k^{3}+(\beta N)^{3}\right)
$$




\subsection{Trust information computation time}

In order to minimize the time required to evaluate the trust profile of a large MANET, the optimal cluster size $\left(k_{\text {optimal }}\right)$ that minimizes the computational complexity used in evaluating the trust profile of all nodes in the network is derived. As shown in Fig. 3, the computational complexity is directly proportional to the computational time required in evaluating the trust profile of all nodes of the MANET.

\subsection{Minimization of computational complexity}

The main objective of the CGTrust scheme is to minimize the computational complexity that results in a minimized network reconfiguration time delay. The optimization statement and constraints are established as below.

$$
\begin{array}{cl}
\underset{k}{\operatorname{minimize}} & C_{\text {total }}(k) \\
\text { subject to } & 0 \leq \beta \leq 0.5, \\
& N, k \in \mathbb{Z}_{>0} .
\end{array}
$$

The total computational complexity in the TA is a function of $k$ in the form of $C_{\text {total }}$, where the computational complexity minimizing optimal number of clusters can be obtained from

$$
\begin{aligned}
k^{\prime} & =\arg \left[\frac{\partial C_{\text {total }}(k)}{\partial k}=0\right] \\
& =\arg \left[-\frac{3\left(N^{3}+(\beta-1)^{3} k^{6}\right)}{k^{4}}=0\right]
\end{aligned}
$$

which results in the following candidate solutions.

$$
k^{\prime}=\left\{\begin{array}{ccc}
\frac{\sqrt{N(1-\beta)}}{1-\beta}, & \frac{-\sqrt{N(1-\beta)}}{1-\beta}, & -\sqrt{\frac{N(i \sqrt{3}+1)}{2(1-\beta)}} \\
\sqrt{\frac{N(i \sqrt{3}+1)}{2(1-\beta)}}, & -\sqrt{\frac{N(i \sqrt{3}-1)}{2(1-\beta)}}, & \sqrt{\frac{N(i \sqrt{3}-1)}{2(1-\beta)}}
\end{array}\right\}
$$

There are 6 solutions of (13), where $N$ is a positive number and the range of $\beta$ is $[0,0.5]$. Considering the fact that

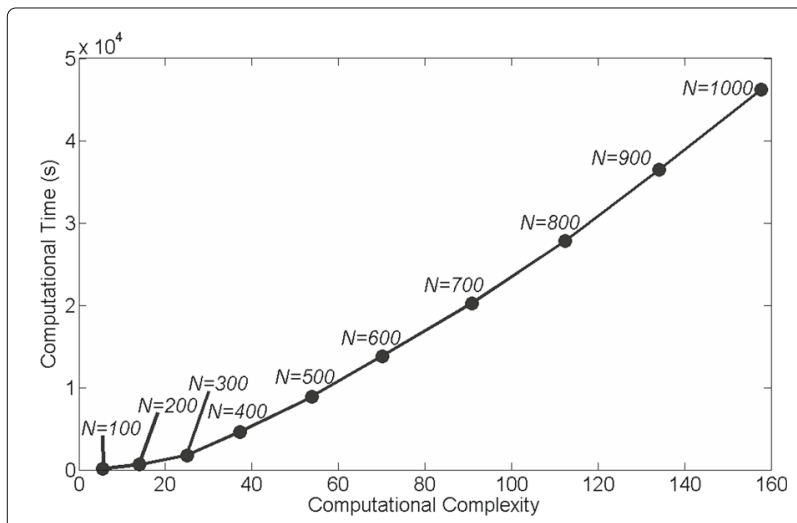

Fig. 3 Relation of computational complexity and time $k$ needs to be a positive integer, the only feasible solution is $\frac{\sqrt{N(1-\beta)}}{1-\beta}$ which is a positive real number. In addition, the second derivative of the objective function is

$$
\frac{\partial^{2}}{\partial k^{2}} C_{\text {total }}(k)=\frac{12 N^{3}}{k^{5}}+6(1-\beta)^{3} k
$$

in which $k^{\prime}=\frac{\sqrt{N(1-\beta)}}{1-\beta}$ results in a positive value of the convex objective function $C_{\text {total }}(k)$. The optimal number of clusters $\left(k_{\text {optimal }}\right)$ has to be a positive integer, and therefore, the nearest integer function (i.e., $\operatorname{Nint}(\cdot))$ is applied to result in (15).

$$
k_{\text {optimal }}=\operatorname{Nint}\left(\frac{\sqrt{N(1-\beta)}}{1-\beta}\right)
$$

Figure 4 presents the $C_{\text {total }}$ profile and the $k_{\text {optimal values }}$ for the range of interest based on $N$ and $k$.

\section{Performance evaluation}

Malicious attack patterns investigated for the FN and FP performance evaluation include the following five attack patterns [2].

- Naive malicious attack (NMA): a malicious node provide improper services with probability $\alpha$. However, it reports its LTOs honestly.

- Collusive rumor attack (CRA): In addition to providing improper services with probability $\alpha$, malicious nodes collude to report false LTOs. Malicious nodes report LTOs of 1 to malicious node and LTOs of 0 to honest nodes.

- Non-collusive rumor attack (NRA): a malicious node can report a false LTO that is opposite to the observed evidence. For example, if an LTO is evaluated as $p$, the malicious node may report $(1-p)$ as the LTO.

- Malicious spy attack (MSA): some malicious nodes misbehave with probability $\alpha$. Other malicious nodes behave honestly. These malicious nodes may collude

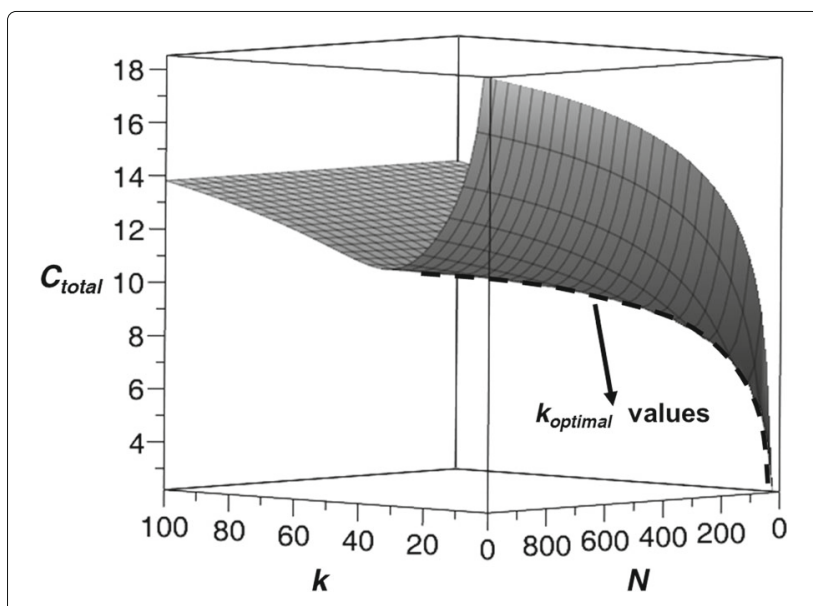

Fig. 4 Minimum points of $C_{\text {total }}\left(k_{\text {optimal }}\right)$ according to $N$ and $k$ 
and report LTOs of 1 to malicious node and LTOs of 0 to honest nodes to confuse the trust and reputation system.

- Conflicting behavior attack (CBA): malicious nodes can collude to confuse the trust and reputation system such as CRA and MSA. However, they misbehave only to some of honest nodes, and report LTOs of 1 to malicious node and LTOs of 0 to honest nodes to confuse the trust and reputation system. This attack causes LTO disagreement among honest nodes, which makes it difficult to find malicious nodes.

CBA is considered the most demanding type of attack because it makes it difficult to distinguish malicious nodes by confusing LTO information of honest nodes with respect to other nodes. For the above reasons, CBA was selected and evaluated.

The simulation based performance analysis of CGTrust and GlobalTrust was conducted using Matlab with $N$ nodes randomly distributed with a uniform density in a $2 \times 2 \mathrm{~km}^{2}$ square area. Simulation parameters were set same to the experiments in [2], where the ratio of malicious nodes was set to 0.3 and every node randomly requests of its neighbor nodes to send a packet (which is multihop relayed) 100 times per minute, and $\beta$ is in the range in $[0,0.5]$. Honest nodes were made to drop packets based on a 0.05 packet error rate (PER) and the detection error probability of the monitoring system was set to 0.05. Each node was made to transmit trust data packets every $30 \mathrm{~s}$ and the TA computes the GRs based on the accumulated data of the past $30 \mathrm{~min}$.

In addition, the probability that a malicious node drops a packet was set to $0.5, \gamma=0.7, \theta=0.7$, and the upper bound probability of FN and FP were set to 0.1 as used in [2].

In the simulation, the TA is not a target of a malicious node. If the TA is infected, trust decisions on network nodes will not be correct. It is assumed that $\mathrm{CH}$ and other nodes can be malicious nodes, based on the restriction that the malicious ratio is not more than 0.5. If the malicious node ratio is greater than 0.5 , the malicious nodes can take control of all the opinions in the network and the trust decision cannot be determined correctly. Although it is assumed that the overall ratio of malicious nodes is less than 0.5 , the proportion of malicious nodes in a cluster is not limited. Therefore, in some clusters, malicious nodes may not properly report to the TA because they have taken control of the cluster.

Figure 5 compares $C_{\text {total }}$ of GlobalTrust and CGTrust based on $\beta$, where it can be observed that $C_{\text {total }}$ of CGTrust decreases to less than 1/1000 compared to GlobalTrust. The results show that CGTrust can significantly reduce the required computations and thereby reduce the network's trust evaluation time. Figure 6 shows the

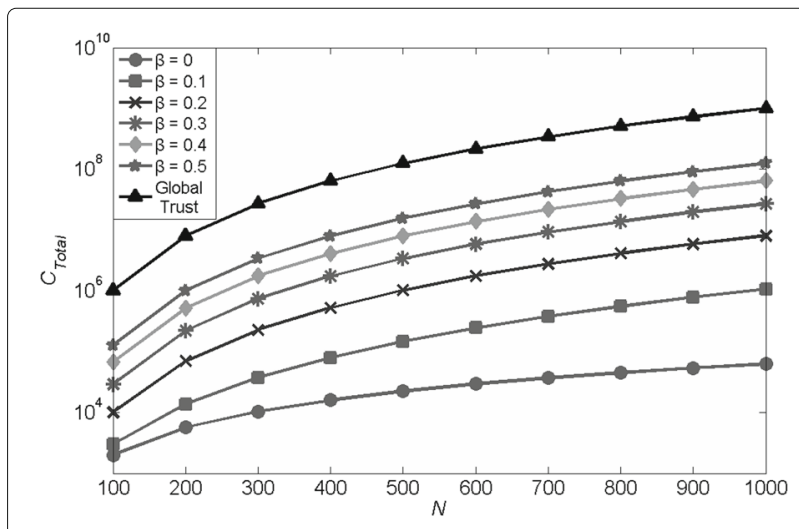

Fig. $5 C_{\text {total }}$ Comparison of GlobalTrust and CGTrust based on $N$ and $\beta$

detection error probability of FN and FP for CGTrust, where for the number of nodes of interest (i.e., $100 \leq N \leq$ 1000), the probability of FN and FP are always lower than the upper bound 0.1. More significantly, the FN probability is always below 0.00012 . In addition to being much faster than GlobalTrust, having a very low FN probability is a very advantageous feature of CGTrust because (compared to FP) FN is a significantly more critical security problem due to the fact that FN represents the probability that a malicious node has been not detected and still remains operational in the network. On the other hand, an erroneous FP decision on a node can be easily corrected by additional checking of the node. Figure 7 investigates influence of changes in malicious node ratios, where the results show that the FN probability of CGTrust is an approximate 0.1 times lower compared to GlobalTrust, while the FP probability of the two are similar. Abrupt changes in the performance can be observed in Figs. 4 and 5 , which are due to the positive integer rounding effect applied to $k_{\text {optimal }}$.

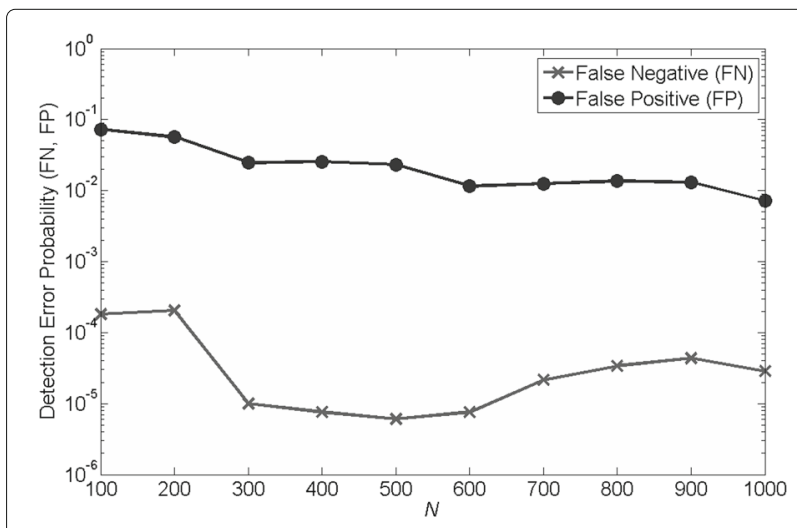

Fig. 6 Detection error probability (FN and FP) of CGTrust 


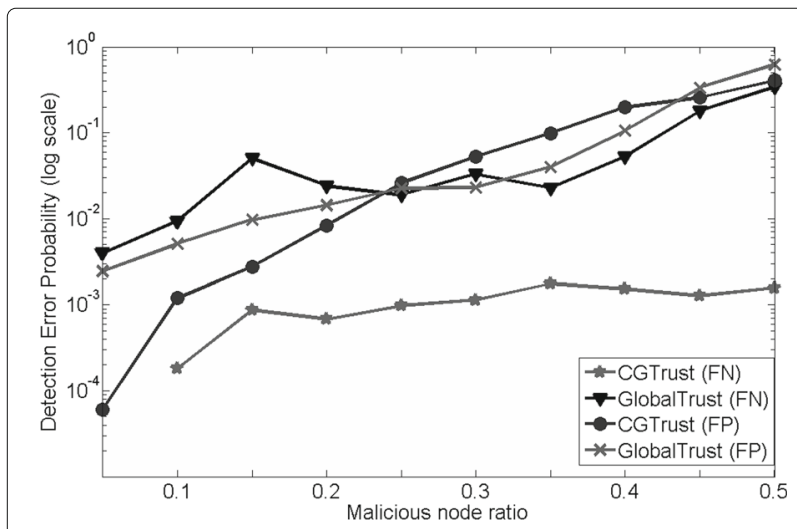

Fig. 7 Detection error probability (FN and FP) comparison of GlobalTrust and CGTrust based on various malicious node ratios

\section{Conclusion}

Mission supportive MANETs require fast updates on node conditions in order to properly support command and control operations. To support this objective, CGTrust was designed to minimize the time required to evaluate the trust profile of a MANET through optimal cluster size control applied to GlobalTrust. The simulation results show that for the number of nodes and malicious node ratios of practical interest (based on $\beta=0.1$ and 0.5 ), CGTrust can be approximately 1000 and 10 times faster compared to GlobalTrust, respectively. In addition, the results also show that the FN probability is approximately 0.1 times lower when CGTrust is used instead of GlobalTrust for the malicious node ratio range of 0.05 to 0.5 .

\section{Acknowledgements}

This work was supported by the ICT R\&D program of MSIT/ITP, Republic of Korea (B0101-17-1276, Access Network Control Techniques for Various loT Services).

\section{Competing interests}

The authors declare that they have no competing interests.

\section{Publisher's Note}

Springer Nature remains neutral with regard to jurisdictional claims in published maps and institutional affiliations.

\section{Author details \\ ${ }^{1}$ School of Electrical and Electronic Engineering, Yonsei University, Seoul, Republic of Korea. ${ }^{2}$ Republic of Korea Air Force, Gyeryong, Republic of Korea.}

Received: 23 February 2017 Accepted: 25 August 2017

Published online: 18 September 2017

\section{References}

1. J-H Cho, A Swami, I-R Chen, A Survey on Trust Management for Mobile Ad Hoc Networks. IEEE Commun. Surv. Tutor. 13(4), 562-583 (2011)

2. X Chen, J Cho, S Zhu, in Proceedings of the IEEE International Conference on Sensing, Communication and Networking (SECON) 2014. GlobalTrust: An Attack-Resilient Reputation System for Tactical Networks, (Singapore, 2014), pp. 275-283

3. K Aberer, Z Despotovic, in Proceedings of the 2001 ACM International Conference on Information and Knowledge Management (CIKM). Managing trust in a peer-2-peer information system, (Atlanta, GA, USA, 2001), pp. 310-317

4. SD Kamvar, MT Schlosser, H Garcia-Molina, in Proceedings of the 2003 ACM International Conference on World Wide Web (WWW). The eigentrust algorithm for reputation management in p2p networks, (Budapest, Hungary, 2003), pp. 640-651

5. L Xiong, L Liu, Peertrust: Supporting reputation-based trust for peer-to-peer electronic communities. IEEE Trans. Knowl. Data Eng. 16(7), 843-857 (2004)

6. S Buchegger, JY Le Boudec, A Robust Reputation System for Mobile ad hoc Networks. Technical Report IC/2003/50, EPFL-DI-ICA, (Lausanne, 2003)

7. Q He, D Wu, P Khosla, in Proceedings of the IEEE Wireless Communications and Networking Conference (WCNC) 2004. Sori: a secure and objective reputation based incentive scheme for ad-hoc networks, (Atlanta, GA, USA, 2004), pp. 825-830

8. WL Teacy, J Patel, NR Jennings, M Luck, Travos: Trust and reputation in the context of inaccurate information sources. Auton. Agent Multi Agent Syst. 12(2), 183-198 (2006)

9. A Jsang, R Ismail, in Proceedings of the Electronic Commerce Conference 2002. The beta reputation system, (Bled, Slovenia, 2002), pp. 2502-2511

10. H Chan, VD Gligor, A Perrig, G Muralidharan, On the distribution and revocation of cryptographic keys in sensor networks. IEEE Trans. Dependable Secure Comput. 2(3), 233-247 (2005)

11. M Raya, MH Manshaei, M Felegyhazi, J-P Hubaux, in Proceedings of ACM Conference on Computer and Communications Security (CCS) 2008. Revocation games in ephemeral networks, (Alexandria, VA, USA, 2008), pp. $199-210$

12. S Reidt, M Srivatsa, S Balfe, in Proceedings of the ACM Conference on Computer and Communications Security (CCS) 2009. The fable of the bees: incentivizing robust revocation decision making in ad hoc networks, (Chicago, IL, USA, 2009), pp. 291-302

13. X Chen, H Patankar, S Zhu, M Srivatsa, J Opper, in Proceedings of the IEEE International Conference on Sensing, Communication and Networking (SECON) 2013. Zigzag: Partial mutual revocation based trust management in tactical ad hoc networks, (New Orleans, LA, USA, 2013), pp. 131-139

14. S Kim, J-M Chung, Message Complexity Analysis of Mobile Ad Hoc Network Address Autoconfiguration Protocols. IEEE Trans. Mobile Comput. 7(3), 358-371 (2008)

\section{Submit your manuscript to a SpringerOpen ${ }^{\circ}$ journal and benefit from:}

- Convenient online submission

- Rigorous peer review

- Open access: articles freely available online

- High visibility within the field

- Retaining the copyright to your article

Submit your next manuscript at $\boldsymbol{\sim}$ springeropen.com 